\title{
The ability of clinical and laboratory findings to predict in-hospital death in patients with thrombotic thrombocytopenic purpura in an internal and emergency medicine department
}

\section{L'impatto delle variabili cliniche e di laboratorio sull'outcome di pazienti con porpora trombotica trombocitopenica in un dipartimento di medicina interna e d'urgenza}

\section{Filippo Pieralli ${ }^{a, *}$, Antonio Mancini ${ }^{a}$, Alberto Camaiti ${ }^{b}$, Giancarlo Berni ${ }^{a}$, Carlo Nozzoli ${ }^{a}$}

\footnotetext{
a Internal and Emergency Medicine Department (Chief: Carlo Nozzoli), Azienda Ospedaliero-Universitaria Careggi, Florence, Italy

${ }^{\mathrm{b}}$ Medicina Interna, USL 6 Livorno, Italy
}

Received 7 October 2010; accepted 21 February 2011

available online 6 April 2011

\section{KEYWORDS \\ Thrombotic thrombocytopenic purpura; hemolytic anemia; lactic acid.}

\begin{abstract}
Summary
Introduction: Thrombotic thrombocytopenic purpura (TTP) is a rare, life-threatening syndrome characterized by microangiopathic anemia, thrombocytopenia, diffuse microvascular thrombosis, and ischemia. It is associated with very low levels of ADAMTS-13. Measurement of ADAMTS-13 levels is used for diagnostic and prognostic purposes, but in every-day clinical practice, this type of analysis is not always readily available. In this retrospective study, we evaluated prognostic value of clinical and laboratory findings in patients with TTP.

Materials and methods: We retrospectively investigated patients with clinically diagnosed TTP treated in a unit of Internal and Emergency Medicine (1996-2007). Clinical and laboratory findings were collected and analyzed in order to assess their ability to predict in-hospital death. Results: Twelve patients were identified (mean age $59 \pm 22$ years; $58 \%$ were women). Five (42\%) died during the hospitalization, and the variables significantly associated with this outcome were: a delay between diagnosis and symptom onset (HR 1.36; 95\% Cl 1.04-1.78; $\mathrm{p}<0.05$ ); a higher severity score (HR 1.48; 95\% Cl 1,23-3.86; $\mathrm{p}<0.05)$; hemodynamic instability with hypotension and/or shock ( $\mathrm{HR} 3.35 ; 95 \% \mathrm{Cl} 3.02-9.26$; $\mathrm{p}<0.01)$; a higher schistocyte count on
\end{abstract}

\footnotetext{
* Corresponding author: Viale Fratelli Rosselli 39 - 50144 Florence, Italy.

E-mail: filpiero@tin.it (F. Pieralli).
} 
blood smear (HR 1.84; 95\%Cl 1.04-3.27; $\mathrm{p}<0.05)$; and higher lactate values $(\mathrm{HR} 1.85 ; 95 \% \mathrm{Cl} 1.08$ 3.16; $\mathrm{p}<0.05)$.

Conclusions: TTP is a rare and potentially fatal disease with protean manifestations. Delayed diagnosis after symptom onset is a major determinant of poor outcome. Hypotension and shock are also prognostically unfavourable. Laboratory evidence of cardiocirculatory compromise (i.e., elevated lactate levels) and extension of the disease process (i.e., schistocyte count $>3$ ) are predictive of in-hospital death, independently of the hemodynamic profile on admission.

(c) 2011 Elsevier Srl. All rights reserved.

\section{Introduction}

Thrombotic thrombocytopenic purpura (TTP) is a rare, lifethreatening familial or acquired syndrome characterized by microangiopathic anemia and thrombocytopenia with diffuse microvascular thrombosis and organ ischemia. It was first described by Moschcowitz in 1925 [1]. Mortality may exceed $90 \%$ if the disease is untreated; however, with the introduction of plasma exchange as a standard of therapy, mortality has been significantly reduced $[2,3]$. The clinical picture is often unrevealing, with subtle presenting symptoms and non-specific laboratory findings that frequently leads to a delay in diagnosis [4]. The pathophysiology of TTP has recently been elucidated. A genetic mutation or the presence of inhibitory antibodies lead to reduced production of ADAMTS13 (A Disintegrin and Metalloprotease with Thrombospondin-1-like domains), an enzyme that cleaves von-Willebrand factor [5], and ultra-large plasmatic multimers of von-Willebrand factor (ULVWF) accumulate. These ULVWF multimers bind efficiently to glycoprotein (GP) lb of platelet GPIb-IX-V receptors, promoting diffuse platelet aggregation with organ ischemia, thrombocytopenia and microangiopathic anemia. Measured levels of ADAMTS13 activity are severely reduced $(<5 \%)$ in TTP relative to several conditions mimicking Moschcowitz disease (sepsis, cancer, heparin-induced thrombocytopenia and hemolytic-uremic syndrome). Measurement of ADAMTS13 activity has been demonstrated to be a useful tool for diagnostic and prognostic purposes, but its clinical application is currently limited by the unavailability of assays in most hospitals [6,7]. In this retrospective study, we sought to describe the clinical characteristics of patients with TTP and to determine the significance of simple and readily available clinical and laboratory tests performed on admission in predicting inhospital mortality.

\section{Materials and methods}

We retrospectively evaluated cases with a first episode of clinically diagnosed TTP, defined by the presence of severe hemolytic microangiopathic anemia and thrombocytopenia without an apparent alternative etiology. All patients were admitted directly from the Emergency Room in the Internal and Emergency Medicine unit of a large, regional, universityaffiliated referral hospital between March 1996 and December 2007. TTP patients were identified according to a clinical algorithm applied to all cases of combined anemia and thrombocytopenia (Fig. 1). All patients with a clinical diagnosis of TTP were admitted to a High Dependency Unit, with continuous monitoring of cardiac frequency, respiratory frequency and sp02; blood pressure was usually monitored non-invasively and intermittently, except in those cases for which an arterial line was inserted. Venous blood samples were drawn to measure common laboratory parameters,

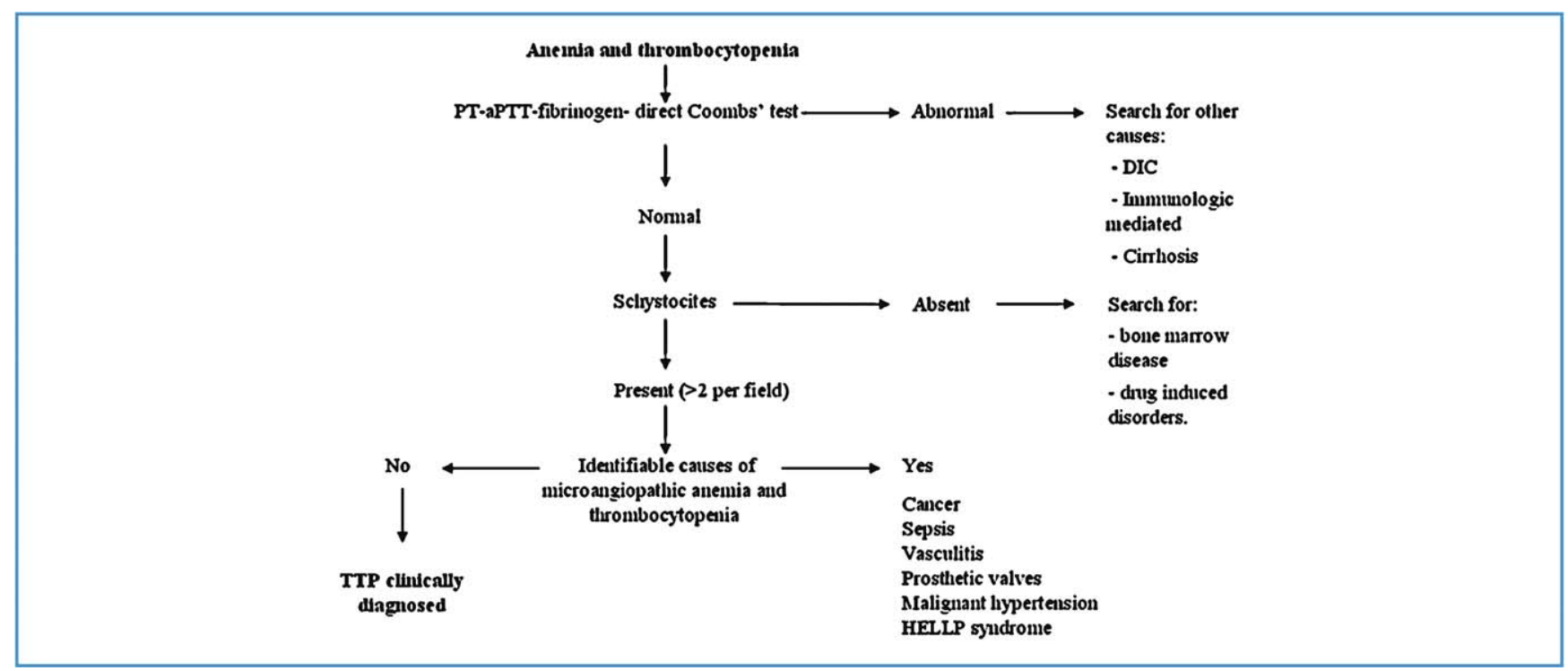

Figure 1 Clinical algorythm to diagnose thrombotic thrombocytopenic purpura (TTP). 
while lactate was measured from an arterial sample (ABL70, Radiometer Medical A/S, Copenhagen, DK). A severity score, based on a work of Rose and Eldor [8], was calculated on presentation based on the following four clinical and laboratory parameters: neurologic findings, impairment of renal function, platelet count, and hemoglobin value. Each parameter was graded from 0 to $2(0=$ no abnormality; $1=$ mild to moderate impairment; 2 =severe impairment). The patient's severity score was the sum of the four variables and ranged from 0 to 8 (most severe). Measurement of ADAMTS-13 activity by ELISA test was introduced at our Hospital in 2006 (TECHNOZYM ${ }^{\circledR}$ ADAMTS-13 Activity ELISA Chromogenic test, Technoclone, Vienna, Austria), so ADAMTS-13 activity detection was available in only three patients. All patients received steroid treatment: either prednisone at $1 \mathrm{mg} / \mathrm{kg} / \mathrm{die}$, or methyl-prednisolone as a $1 \mathrm{~g}$ single bolus per day for three consecutive days, followed by prednisone $1 \mathrm{mg} / \mathrm{kg} / \mathrm{die}$; the choice of steroid regimen was made by the single attending physician. Fresh-frozen plasma was administered to all patients. Plasma exchange was started as soon as possible; the exchanged plasma volume was determined by the transfusion medicine physician depending on clinical and hemodynamic profile. A patient was considered to be in remission when the platelet count reached at least $100.000 / \mathrm{mm}^{3}$.

\section{Statistical analysis}

All variables are expressed as means and percentages. For normally distributed continuous variables, the student T-test was used; a $p<0.05$ was considered statistically significant. For non-continuous variables, the Fisher's exact test was used, and a $\mathrm{p}<0.05$ was considered statistically significant. The hazard ratio was calculated with the Cox regression analysis model. The SPSS statistical software package, $10^{\text {th }}$ edition, was used for statistical analysis (SPSS, Chicago, III., USA).

\section{Results}

During the study period, twelve patients with TTP were identified. The main clinical characteristics and laboratory findings are presented in Table 1. Females represented $58 \%$ of the study population and were younger than males $(50.5$ vs. 67 years; $p<0.005)$. Fluctuating neurological disorders and fever were the most prevalent signs; renal failure (i.e., creatinine $\geq 1.4 \mathrm{mg} / \mathrm{dL}$ ) was present in four patients, and purpura was present two patients. The elapsed time from symptom onset to diagnosis was varied (3-36 days). All patients received high-dose steroid therapy: six patients were initially treated with pulse bolus of methyl-prednisolone; five patients were treated with prednisone $1 \mathrm{mg} / \mathrm{kg}$. All patients received urgent treatment with fresh-frozen plasma; the mean daily infused plasma volume was $1460 \pm 175 \mathrm{~mL}$, with a mean duration of treatment of $8.2 \pm 1.3$ days. The mean daily infusion of fresh-frozen plasma did not account for any difference in survival $(1380 \pm 95$ vs. $1490 \pm 100, p=$ n.s. $)$. The total duration of treatment was shorter in the group of patients who died $(4.6+2$ vs. $9.6+1.2 ; p<0.05)$. Plasma exchange was performed in nine patients $(75 \%)$. Five patients $(42 \%$ of
Table 1 Presenting clinical features and laboratory test results at the time of diagnosis of 12 patients with TTP. Data in parentheses are expressed as percentage or range of values.

\begin{tabular}{ll}
\hline $\begin{array}{l}\text { Clinical characteristics } \\
\text { Age (years) }\end{array}$ & $59 \pm 22(37-85)$ \\
Female & $7(58 \%)$ \\
Presenting symptoms & \\
$\quad \begin{array}{l}\text { Fluctuating neurological } \\
\text { disorders }\end{array}$ & $9(83 \%)$ \\
Fever & $9(83 \%)$ \\
Weakness & $10(91 \%)$ \\
Abdominal pain & $1(9 \%)$ \\
Hematuria & $2(18 \%)$ \\
Purpura & $2(18 \%)$ \\
Renal failure & $4(36 \%)$ \\
Delay from symptoms onset & $6(3-36)$ \\
$\quad$ to diagnosis (days) & \\
Laboratory test results & \\
Platelet count (cells/ $\mu \mathrm{L})$ & $24300(9000-63,000)$ \\
Hemoglobin (g/dL) & $7.2(4.5-11.7)$ \\
White Blood Cells count & $11000(11000-19000)$ \\
$\quad$ (cells/ $\mu L)$ & $3(2-6)$ \\
Average schistocytes per field & $1.5(0.8-2.1)$ \\
Creatinine (mg/dL) & $1005(726-1429)$ \\
LDH (Ul/L) & $3.6(0.9-8.1)$ \\
Lactate (mmol/L) & \\
\hline
\end{tabular}

* LDH: Lactate dehydrogenase.

patients; three females) died during the in-hospital stay; of these five, two patients did not receive plasma exchange therapy due to severe hemodynamic compromise. Plasma exchange procedures did not account for any difference in survival. Post-mortem histological examination, performed in four patients, confirmed the diagnosis of TTP. ADAMTS-13 activity was tested in only three patients (see methods); of these $<5 \%$ confirmed the clinical diagnosis of TTP. Moreover, the results were available many days after the admission and therefore could not be used in clinical decisions. In Table 2, the clinical features and laboratory findings are described according to in-hospital outcome. In the hospital, the death rate was $42 \%$, and the following variables were significantly associated with a poor outcome: a delay in diagnosis of TTP from recorded symptom onset (HR 1.36; $95 \% \mathrm{Cl}$ 1.04-1.78; $\mathrm{p}<0.05)$, a higher severity score (HR 1.48; $95 \% \mathrm{Cl} 1,23-3.86 ; \mathrm{p}<0.05$ ), hemodynamic instability (defined by the presence of hypotension and/or shock) (HR 3.35; 95\% Cl 3.02-9.26; $\mathrm{p}<0.01)$, a higher schistocyte count on the blood smear (HR 1.84; 95\%Cl 1.04-3.27; $\mathrm{p}<0.05$ ), and higher plasma lactate concentration values (HR $1.85 ; 95 \% \mathrm{Cl}$ 1.08-3.16; $\mathrm{p}<0.05)$.

Long-term follow-up (range 11-63 months) after hospital discharge was available for four surviving patients (57\%). Two of the four patients had relapses and required repeated plasma exchange and long-term immunosuppressive therapy (cyclophosphamide and azatioprine, and splenectomy in one); they are currently patients in the Hematology Unit of this Hospital. 
Table 2 Clinical features and laboratory findings on hospital admission in 12 patients with TTP according to in-hospital outcome. Data in parentheses are expressed as percentage or range of values.

\begin{tabular}{|c|c|c|c|}
\hline & \multicolumn{3}{|c|}{ In-hospital follow-up } \\
\hline & Death (5 patients; 42\%) & Discharged (7 patients; 58\%) & $p$ value \\
\hline \multicolumn{4}{|l|}{ Clinical characteristics } \\
\hline Age (years) & $59 \pm 18$ & $57 \pm 12$ & n.s. \\
\hline Male & 2 & 3 & n.s. \\
\hline Female & 3 & 4 & n.s. \\
\hline Length of stay (days) & $8.3 \pm 3.5$ & $14 \pm 2.8$ & n.s. \\
\hline \multicolumn{4}{|l|}{ Presenting symptoms } \\
\hline Neurological disorders & 5 & 5 & n.s. \\
\hline Fever & 4 & 5 & n.s. \\
\hline Abdominal pain & 0 & 2 & n.s. \\
\hline Purpura & 2 & 0 & n.s. \\
\hline Renal failure & 2 & 2 & n.s. \\
\hline Severity score & $5.6+0.9$ & $3.3+1.0$ & $<0.05$ \\
\hline Delay from symptoms onset to diagnosis (days) & $18 \pm 13$ & $8 \pm 5$ & $<0.05$ \\
\hline \multicolumn{4}{|l|}{ Hemodynamic features } \\
\hline Heart rate (bpm) & $121 \pm 22$ & $86 \pm 20$ & $<0.01$ \\
\hline SBP (mmHg) & $92 \pm 13$ & $113 \pm 30$ & $<0.05$ \\
\hline Respiratory rate $\left(\min ^{-1}\right)$ & $21 \pm 6$ & $17 \pm 5$ & n.s. \\
\hline \multicolumn{4}{|l|}{ Laboratory test results } \\
\hline Platelet count (cells/ $\mu \mathrm{L}$ ) & $16.600 \pm 11.800$ & $38.000 \pm 15.300$ & n.s. \\
\hline Hemoglobin $(\mathrm{g} / \mathrm{dL})$ & $7.8 \pm 2.0$ & $7.7 \pm 1.8$ & n.s. \\
\hline Average number of schistocytes per field & $5.4 \pm 1.8$ & $2.5 \pm 0.6$ & $<0.05$ \\
\hline Creatinine (mg/dL) & $1.64 \pm 0.4$ & $1.16 \pm 0.2$ & n.s. \\
\hline LDH $(\mathrm{UI} / \mathrm{L})^{*}$ & $1101 \pm 210$ & $787 \pm 214$ & n.s. \\
\hline Lactate (mmol/L) & $6.3 \pm 1.5$ & $1.3 \pm 0.4$ & $<0.01$ \\
\hline
\end{tabular}

\section{Discussion}

TTP is a rare familial or sporadic microangiopathic disease with an estimated annual incidence of 3.7 to 11 cases per million people in the U.S. $[9,10]$. Despite recent advances in understanding the pathophysiology of the disease and the increased availability of tests for ADAMTS-13, the diagnosis of TTP in practical settings is still usually made on clinical grounds through the exclusion of other possible disorders inducing anemia and thrombocytopenia (Fig. 1). In this retrospective study, we describe the clinical characteristics of patients with TTP and the significance of simple and readily available clinical and laboratory findings in predicting in-hospital mortality of patients with clinically diagnosed TTP. A combination of symptoms and signs (fever, neurological abnormalities, renal failure, thrombocytopenia and microangiopathic hemolytic anemia) termed "the pentad" has long been recognized as characteristic of TTP, but is rarely observed $[11,12]$. In this study, ambiguous and nonspecific disturbances such as fever and weakness were prevalent. There was a high occurrence of neurological disorders (83\%), ranging from fluctuating focal deficits mimicking ischemic attacks to coma and generalized seizures. It is noteworthy that neurological symptoms were the clinical manifestations that prompted hospital admission. In general, these results correspond with data recorded in large registries [9]. Purpura, despite being the name of the disease, rarely manifested in our patients, and clinically significant hemorrhages were not recorded. Renal failure, which, according to the recent literature, is reported in only a minority of patients, was moderately prevalent in our patient sample $[9,11,12]$. In our study, the etiology of TTP remained unknown in most of the patients; a strong relationship between ticlopidine treatment and TTP was suspected in two patients; estroprogestinic therapy and post-partum disease were suspected in one case. These data are in accordance with the literature: a causative agent of TTP remains unknown in more than $80 \%$ [13]. The time range from symptom onset to diagnosis was wide (3-36 days). Since TTP is an aggressive disease, it is not surprising that those patients who had a delayed diagnosis had a worse outcome than those patients with an earlier diagnosis. Frequent misleading diagnoses made before hospital admission included depression, anxiety, and suspected thyroid disorders, especially in young patients. The mortality rate was high (42\%) despite intensive treatment, fresh-frozen plasma transfusion and every effort to provide plasma exchange therapy. The difference in the duration of plasma infusion between patients who died and those who survived was due to patient death rather than to discontinuation of treatment. The mortality rate in this study was higher than that reported in randomized controlled trials of plasma exchange in TTP, but a wide variability is reported in those papers [3]. This is not surprising because of the limited number of patients included in this retrospective 
study and the acute clinical setting where the investigation was carried out.

The hemodynamic profile on admission was predictive of a poor prognosis; hypotension and shock were present in three patients and resulted in a three-fold increase in relative risk of death. It is important to note that two of these patients did not receive plasma exchange therapy due to severe hemodynamic compromise and refractory hypotension after resuscitation efforts. A severity score according to Rose and Eldor [8] was calculated for each patient, and higher values were predictive of in-hospital death, with a $48 \%$ increase in relative risk for each incremental point in the score. The score can easily be calculated; however, to our knowledge this scoring has not been validated, and the subjective interpretation of qualitative data (i.e., mild, moderate or severe abnormalities) is a limitation on its usefulness. The number of schistocytes in the blood smear was predictive of death, with an $84 \%$ increase in relative risk for each schistocyte counted. Plasma lactate levels were predictive of a worse outcome independent of hemodynamic status, with a relative risk of death of $85 \%$. The relationships among schistocyte count, lactate levels, and death may reflect diffuse involvement of microvascular circulation. It is noteworthy that lactate level, which is readily measurable using modern blood gas analyzers, is a useful tool for triage of those patients who present with with occult hemodynamic compromise despite having normal or near-normal blood pressure values. This pair of symptoms should warrant a timely and aggressive treatment approach despite a normal hemodynamic profile.

The main limitations of this study are the retrospective nature of the investigation and the limited number of patients observed. Nevertheless, it is necessary to point out that these limits are frequently encountered when rare diseases, such as TTP, are considered. Another possible limitation of the study is that of ADAMTS-13 levels were measured in only three patients however, in the real clinical context, the availability of ADAMTS-13 is limited to selected centers [14]. Moreover, there are discrepancies among assay techniques $[14,15]$ which limit the routine clinical application of such methods. For these reasons, ADAMTS-13 measurement is not a routinely available aid in acute care and therefore cannot influence real-time clinical decision making or treatment strategy $[16,17]$.

TTP is a rare disease with a high lethality rate when untreated. It is difficult to diagnose with a high degree of certainty, particularly in the acute care setting. The clinical manifestations of TTP are protean and can be confounded with more benign syndromes until the late stage of the disease. Delay from symptom onset to diagnosis is a major determinant of poor outcome. Laboratory signs of occult circulatory compromise (e.g., elevated plasma lactate levels) and of progression of the disease (e.g., schistocyte count) are determinants of prognosis independent of the hemodynamic profile on presentation, offering a potential tool for early risk stratification.

\section{Conflict of interest statement}

The authors have no conflict of interest.

\section{References}

[1] Moschcowitz E. An acute febrile pleiochromic anemia with hyaline thrombosis of the terminal arterioles and capillaries: an undescribed disease. Arch Intern Med 1925;36(1):89-93.

[2] Rock GA, Shumak KH, Buskard NA, et al. Comparison of plasma exchange with plasma infusion in the treatment of thrombotic thrombocytopenic purpura. N Engl J Med 1991; 325:393-7.

[3] Brunskill SJ, Tusold A, Benjamin S, Stanworth SJ, Murphy MF. A systematic review of randomized controlled trials for plasma exchange in the treatment of thrombotic thrombocytopenic purpura. Transfus Med 2007 Feb;17:17-35.

[4] Eldor A. Thrombotic thrombocytopenic purpura: diagnosis, pathogenesis and modern therapy. Baillière's Clinical Haematology 1998;11:475-514.

[5] Furlan M, Robles R, Galbusera M, et al. Von Willebrand factorcleaving protease in thrombotic thrombocytopenic purpura and the hemolytic-uremic syndrome. N Engl J Med 1998;339: 1578-84.

[6] Bianchi V, Robles R, Alberio L, Furlan M, Lämmle B. Von Willebrand factor-cleaving protease (ADAMTS13) in thrombocytopenic disorders: a severely deficient activity is specific for thrombotic thrombocytopenic purpura. Blood 2002;100:710-3.

[7] Starke R, Machin S, Scully M, Purdy G, Mackie I. The clinical utility of ADAMTS13 activity, antigen and autoantibody assays in thrombotic thrombocytopenic purpura. Br J Haematol 2007 Feb;136:649-55.

[8] Rose M, Eldor A. High incidence of relapses in thrombotic thrombocytopenic purpura. Clinical study of 38 patients. Am J Med 1987;83:437-44.

[9] George JN, Vesely SK, Terrell DR. The Oklahoma Thrombotic Thrombocytopenic Purpura-Hemolytic Uremic Syndrome (TTPHUS) Registry: a community perspective of patients with clinically diagnosed TTP-HUS. Semin Hematol 2004;41:60-7.

[10] Terrell DR, Williams LA, Vesely SK, Lammle B, Hovinga JA, George JN. The incidence of thrombotic thrombocytopenic purpura-hemolytic uremic syndrome: all patients, idiopathic patients, and patients with severe ADAMTS-13 deficiency. J Thromb Haemost 2005;3:1432-6.

[11] George JN. Thrombotic thrombocytopenic purpura. N Engl J Med 2006;354:1927-35.

[12] Moake JL. Thrombotic microangiopathies. N Engl J Med 2002;347:589-600.

[13] Tsai HM. Thrombotic Thrombocytopenic Purpura: A Thrombotic Disorder Caused by ADAMTS13 Deficiency. Hematol Oncol Clin N Am 2007;21:609-32.

[14] Mannucci PM. Thrombotic thrombocytopenic purpura and the hemolytic uremic syndrome: much progress and many remaining issues. Hematologica 2007;92:878-80.

[15] Tsai HM, Lian EC. Antibodies to von Willebrand factor-cleaving protease in acute thromboticthrombocytopenic purpura. $\mathrm{N}$ Engl J Med 1998;339(22):1585-94.

[16] Vesely SK, George JN, Lammle B, et al. ADAMTS13 activity in thrombotic thrombocytopenic purpura-hemolytic uremic syndrome: relation to presenting features and clinical outcomes in a prospective cohort of 142 patients. Blood 2003; 102:60-8.

[17] Ferrari S, Scheiflinger F, Rieger M, et al., for the French Clinical and Biological Network on Adult Thrombotic Microangiopathies. Prognostic value of anti-ADAMTS13 antibody features (Ig isotype, titer, and inhibitory effect) in a cohort of 35 adult French patients undergoing a first episode of thrombotic microangiopathy with undetectable ADAMTS13 activity. Blood 2007;109: 2815-22. 